\title{
UNIQUENESS OF FACTORIZATION OF CERTAIN ENTIRE FUNCTIONS
}

\author{
BY KAZUNARI SAWADA
}

Introduction. For a meremorphic function $F(z)$ in the plane $(|z|<+\infty)$, the representation:

$$
F(z)=f \quad g(z)=f(g(z))
$$

is called a factorization of $F(z)$, where / and $g$ are meromorphic functions ( $g$ is entire, if / is transcendental). And then / is called left-factor and $g$ is called right-factor of $F$. $F$ is called to be prime, if, for every factorization, we can always deduce that either / or $g$ is linear. We state that two factorizations :

$$
\begin{aligned}
F(z) & =f_{1} \circ f_{2} \circ \cdots \circ f_{n} \\
& =g_{1} \circ g_{2} \circ \cdots \circ g_{m}
\end{aligned}
$$

are equivalent, if $n=m$ and there exist linear functions $T_{\jmath}(1 \leqq j \leqq n-1)$ such that

and

$$
f_{1}=g_{1} \circ T_{1}, \quad f_{\jmath}=T_{\jmath-1}{ }^{-1} \circ g_{j} \circ T_{\jmath} \quad(2 \leqq \jmath \leqq n-1),
$$

$$
f_{n}=T_{n-1}^{-1} \circ g_{n}
$$

An entire function $F$ is called uniquely factorizable, if all the factorizations into non-linear prime entire functions are equivalent to each other.

Urabe [8] proved the following

THEOREM A. $F(z)=\left(z+h\left(e^{z}\right)\right) \circ\left(z+Q\left(e^{z}\right)\right)$ is uniquely faciorizable, where his a non-constant entire function, $h\left(e^{z}\right)$ is of finite order and $Q$ is a non-constant polynomial.

We have many functions which are uniquely factorizable as its corollaries. Still there are several functions whose unique factorizablity cannot be proved by Theorem A. For example,

$$
\begin{aligned}
& F(z)=\left(z+e^{z}\right) \cdot\left(z+\frac{1}{e^{z}}\right), \\
& F(z)=\left(z+e^{z}\right) \cdot(z+\sin (-i z))
\end{aligned}
$$

Received November 1, 1991 Revised June 4, 1992. 


$$
=\left(z+e^{z}\right) \cdot\left(z+\frac{e^{z}-e^{-z}}{2 i}\right)
$$

and so on.

In this paper we shall prove the following

THEOREM. Let $R_{j}(w)(j=1,2)$ be non-constant rational functions having at most two poles at $w=0$ and $w-\infty$. Then

$$
F(z)=\left(z+R_{1}\left(e^{z}\right)\right) \cdot\left(z+R_{2}\left(e^{2}\right)\right)
$$

is uniquely factorizable.

As an easy application of this theorem we have immediately that above functions are uniquely factorizable.

§ 1. Some lemmas. We shall use the following symbols:

$$
\begin{aligned}
& M_{F}(r)=M(r, F)=\operatorname{Max}_{|z|=r}|F(z)| \\
& \rho(F)=\limsup _{r \rightarrow \infty} \frac{\log \frac{\log }{\log } \underline{M_{F}} \underline{r}(r)}{}
\end{aligned}
$$

for an entire function $F$. And we shall use Nevanlinna's notations such as $\mathrm{T}(\mathrm{r}, F), m(r, \mathrm{~F})$ and $N(r, a, F)$.

LEMMA 1 (Urabe [8]). Let $J(b)=\{F(z)=c z-H(z) ; H(z)$ is an entire periodic fnnction with period $b(\neq 0)$ and $c$ is a non-zero constant $\}$. And let $F \in J(b)$ and $F(z)=f\left(g(z)\right.$ with non-linear enture functions $f$ and $g$, then $f \in J\left(b^{\prime}\right)$ for some $b^{\prime} \neq 0$ and $g \in J(b)$. Further $b^{\prime}=c_{2} \cdot b$, if $g(z)=c_{2} \cdot z+H_{2}(z)$.

LEMMA 2 (Urabe [8]). Let

$$
F(z)=\left(z+H_{1}(z)\right) \cdot\left(z+H_{2}(z)\right)
$$

where $H_{1}, H_{2}$ (非onstant) are periodic entire functions with period $2 \pi i$ and $\rho\left(H_{1}\right)$ $<+\infty$ and $\mathrm{H}_{2}$ is of exponential type. And let $F(z)=f(g(z))$ with non-linear entire functions $f$ and $g$. Then $g$ is of exponential type.

We recall that $g$ is of exponential type, if $\rho(g) \leqq 1$ and

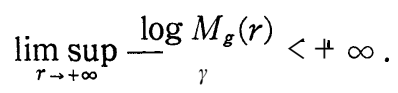

LEMMA 3 (Urabe [8]). Let $H(z)$ (\#constant) be a periodic entire function with period $2 \pi i$ and of exponential type. Then there exist a rational function $R(w)$ with at most two poles at $w=0$ and $w-o o$ such that $H(z) \equiv R\left(e^{z}\right)$.

LEMMA 4 (Ogawa [4]). Let $h(w)$ be single-valued and regular in $0<|w|<\infty$. 
$/ / h\left(e^{z}\right)$ is of finite order, then $h(w)$ is of order zero around $w=0$ and $w=\infty$.

In general, if $h(w)$ is regnlar in $0<|w|<\infty$, there exist two entire fnnctions $h_{j}(w)(j=1,2)$ such that

$$
h(w)=h_{1}(w)+h_{2}\left(\frac{1}{w}\right) .
$$

The above lemma 4 suggests $\rho\left(h_{j}\right)=0$ for $j=1,2$.

LEMMA 5. Let $F(z)$ be the same function in the theorem. And let $F(z)=$ $f(g(z))$ with an entire function $f$ and $g(z)=z+Q\left(e^{z}\right)$, where $Q(w)$ is a rational function with at most two poles at $w=0, \infty$. Then $\rho(f)<+\infty$.

Proof. By Pólya's result,

$$
M_{F}(r) \geqq M_{f}\left(d \cdot M_{g}\left(\frac{r}{2}\right)\right) \quad\left(r \geqq r_{0}\right)
$$

for some positive constant $d$. And by the form of $F$, there exists a positive constant $K$ such that

$$
M_{F}(r) \leqq e^{e^{K \cdot r}}
$$

for any $r \geqq r_{1}$. Further for any $\varepsilon>0$, there exist $r_{2}(>0)$ and some natural number $c$ such that

$$
e^{c / 2 \cdot r-\varepsilon} \leqq M_{\mathbf{g}}\left(\frac{r}{2}\right) \leqq e^{c / 2 \cdot r+\varepsilon}
$$

for $r \geqq r_{2}$.

Therefore, there exists $R_{0}(>0)$ such that

$$
M_{f}(R) \leqq \exp \left[\left(e^{\varepsilon} \cdot \frac{\pi}{d}\right)^{2 K / c}\right]
$$

for $R \geqq R_{0}$. It means that $p(f)<+\infty$.

q. e. d.

§3. Proof of theorem. By the assumption of theorem,

$$
F(z)=z+R_{2}\left(e^{z}\right)+R_{1}\left[e^{z+R_{2}\left(e^{z}\right)}\right] .
$$

Here the function $R_{2}\left(e^{z}\right)+R_{1}\left[e^{z+R_{2}\left(e^{z}\right)}\right]$ is a periodic function with period $2 \pi i$. By lemma 1, if

$$
F(z)=f(g(z))
$$

with non-linear entire functions / and $g$, then

$$
f(z)=c_{1} \cdot z+H_{1}(z), \quad g(z)=c_{2} \cdot z+H_{2}(z)
$$

where $H_{1}, H_{2}$ are periodic with period $2 \pi c_{2} i$, $2 \pi i$ respectively. Substituting these into (1), we have $c_{1} \cdot c_{2}=1$ and hence 
and

$$
f\left(c_{2} \cdot z\right)=c_{1} c_{2} z+H_{1}\left(c_{2} \cdot z\right)=z+H_{1}\left(c_{2} \cdot z\right)
$$

$$
\frac{1}{c_{2}} \cdot g(z)=z+\frac{1}{c_{2}} \cdot H_{2}(z)
$$

belong to $J(2 \pi i)$. Therefore, without loss of generality, we may assume that

$$
\left\{\begin{array}{l}
f(z)=z+H_{1}(z) \\
g(z)=z+H_{2}(z)
\end{array}\right.
$$

where $H_{j}(j=1,2)$ are periodic entire functions with period $2 \pi i$. Further, in general, a periodic entire function with period $2 \pi i$ is represented as $h\left(e^{z}\right)$ with some regular function $h(w)$ in $0<w \mid<+\infty$. Hence

$$
\left\{\begin{array}{l}
f(z)=z+h_{1}\left(e^{z}\right) \\
g(z)=z+h_{2}\left(e^{z}\right)
\end{array}\right.
$$

where $h_{j}(w)$ are regular in $0<|w|<+\infty(j=1,2)$.

Since $\rho\left(R_{1}\left(e^{z}\right)\right)=1<+\infty$ and $R_{2}\left(e^{z}\right)$ is of exponential type, $g$ must be of exponential type by lemma 2 . And then $h_{2}$ must be a rational function by lemma 3. By (1) and (2), we have

$$
h_{2}\left(e^{z}\right)+h_{1}\left[e^{z} \cdot e^{h_{2}\left(e^{z}\right)}\right]=R_{2}\left(e^{z}\right)+R_{1}\left[e^{z} \cdot e^{R_{2}\left(e^{z}\right)}\right] .
$$

Now we put $w=e^{z}$. Then

$$
h_{2}(w)-R_{2}(w)=-h_{1}\left[w \cdot e^{h_{2}(w)}\right]+R_{1}\left[w e^{R_{2}(w)}\right] .
$$

This gives a key of our proof of this theorem. By the above investigation, we assume that

$$
\begin{aligned}
R_{j}(w) & =\left(a_{N_{\jmath}}{ }^{\prime} \cdot w^{N_{\jmath}}+\cdots+a_{0}^{j}\right)+\left(a_{-1}{ }^{\jmath} \cdot \frac{1}{w}+\cdots+a_{-M_{\jmath}}{ }^{\jmath} \cdot \frac{1}{w^{M \jmath}}\right) \\
& =R_{\jmath}{ }^{+}(w)+R_{\jmath}{ }^{-}(w) \quad(j=1,2), \\
h_{2}(w) & =\left(b_{n_{2}}{ }^{2} \cdot w^{n_{2}}+\cdots+{b_{0}}^{2}\right)+\left(b_{-1}{ }^{2} \cdot \frac{1}{w}+\cdots+b_{-m_{2}}{ }^{2} \cdot \frac{1}{w^{m^{2}}}\right) \\
& =h_{2}{ }^{+}(w)+h_{2}{ }^{-}(w) .
\end{aligned}
$$

Similarly we write

$$
h_{1}(w)=h_{1}{ }^{+}(w)+h_{1}{ }^{-}(w),
$$

where in this case both $h_{1}{ }^{+}(w)$ and $h_{1}{ }^{-}(1 / w)$ are entire functions. By lemma 5 , $\rho(f-z)=\rho(f)<+\infty$. And by lemma $4, \rho\left(h_{1}{ }^{+}\right)=\rho\left(h_{1}{ }^{-}(1 / w)=0\right.$.

In the following we shall prove that $h_{1}$ must be a rational function. Now we assume that $h_{1}{ }^{+}$is a transcendental function. Then we will show that $\mathrm{tt}_{2} \wedge \Lambda / 2$ as follows. As noted above, $\rho\left(h_{1}{ }^{+}\right)=0$, and hence by $\cos \pi \rho$-theorem, 
for any $\varepsilon(>0)$, there exists an unbounded sequence of positive real numbers $\left\{r_{n}\right\}$ such that

$$
m_{h_{1}}\left(r_{n}\right) \geqq M_{h_{1}+}\left(r_{n}\right)^{1-\varepsilon} \quad(n=1,2, \cdots),
$$

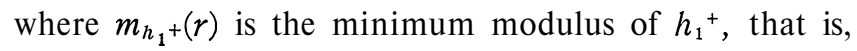

$$
\min _{\mid w_{\mid}=r}\left|h_{1}{ }^{+}(w)\right| \text {. }
$$

Here assuming $n_{2}<N_{2}$, we consider the following equation:

$$
R_{2}{ }^{+}(w)=2 \pi t i \quad(t \in R, t \neq 0) .
$$

As is well-known, the set of roots of equation (5) tend to $w-\infty$ as $|t| \rightarrow \infty$ and possesses $2 N_{2}$ lines:

$$
\begin{aligned}
& \arg w=\frac{1}{N_{2}} \operatorname{Arg}\left(\frac{i}{a_{N_{2}}{ }^{2}}\right)+\frac{1}{N_{2}} 2 \jmath \pi \quad\left(\jmath=, 0,1, \cdots, N_{2}-1 ; \text { as } t \rightarrow+\infty\right) \\
& \arg w=\frac{1}{N_{2}} \operatorname{Arg}\left(\frac{i}{a_{N_{2}}{ }^{2}}\right)+\frac{1}{N_{2}} \cdot(2 j+1) \pi \quad\left(\jmath=0,1, \cdots, N_{2}-1 ; \text { as } t \rightarrow-\infty\right)
\end{aligned}
$$

as asymptotic lines. If $n_{2}>0$, then (because of $n_{2}<N_{2}$ ), among these $2 N_{2}$ lines, we have a line, say /, on which

$$
\operatorname{Re}\left[b_{n_{2}}{ }^{2} \cdot e^{n_{2} \theta}\right]>0 \quad\left(z=r \cdot e^{i \theta} \in l\right) .
$$

And there exists a subset (continuity) $\{w(t)\}$ of roots of (5) such that

$$
R_{2}{ }^{+}(w(t))=2 \pi i t
$$

and further $\{w(t)\}$ possesses the line $l$ as asymptotic line. Therefore by $R_{2}(w(t))$ $=R_{2}{ }^{+}(w(t))+o(1)$,

$$
\left|e^{R_{2}(w(t))}\right| \longrightarrow 1 \quad(\text { as } t \rightarrow+\infty, \text { or as } t \rightarrow-\infty)
$$

and further, there exists some constant $L(>0)$ such that

$$
\mid e^{h_{2}(w(t))}>e^{L \cdot|w(t)|^{n_{2}}} \quad \text { as }|t| \rightarrow+\infty
$$

by the assumption of $\{w(t)\}$. Here, consider a sequence $\left\{t_{n}\right\}$ of real numbers such that

$$
\left|w\left(t_{n}\right) e^{h_{2}\left(w\left(t_{n}\right)\right)}\right|=r_{n} .
$$

Then by (3), (4), (6), (7) and maximum modulus principle, we have

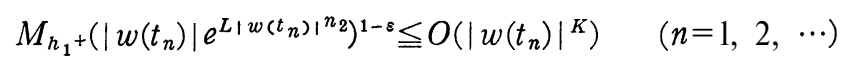

for some constant $K$. Since $h_{1}{ }^{+}$is assumed to be transcendental, this leads us to a contradiction. Hence $n_{2} \geqq N_{2}$. Now let us note that, even if $n_{2}=0$, the above inequality (8) can be shown to be valid without using the special line / 
and hence we get to the same conclusion.

Similarly, if $h_{1}{ }^{-}$is transcendental, then we can prove $m_{2} \geqq M_{2}$.

Since $h_{2}$ is non-constant, $n_{2}>0$ or $\mathrm{ra}_{2}>0$. Without loss of generality, we may assume $n_{2}>0$.

Let, for a sufficiently small $\delta>0\left(\left|b_{n_{2}}{ }^{2}\right| / 2>\delta\right)$,

$$
\begin{aligned}
& O_{1}=\left\{z=r \cdot e^{i \theta} ; \operatorname{Re}\left(b_{n_{2}}{ }^{2} \cdot e^{i n_{2} \theta}\right)>\delta\right\}, \\
& O_{2}=\left\{z=r \cdot e^{i \theta} ; \operatorname{Re}\left(b_{n_{2}}{ }^{2} \cdot e^{i n_{2} \theta}\right)<-\delta\right\} .
\end{aligned}
$$

By $h_{2}(w)=b_{n_{2}}{ }^{2} \cdot w^{n_{2}} \cdot\left(1+o(1)(|w| \rightarrow+\infty)\right.$, it is noted that the function $h_{1}{ }^{+}\left(w e^{h_{2}(w)}\right)$ is bounded in $O_{2} \cap\left\{|w|>R_{0}\right\}$ and $h_{1}{ }^{-}\left(w e^{h_{2}(w)}\right)$ is bounded in $O_{1} \cap\left\{|w|>R_{0}\right\}$. By (3),

$$
M\left(r, R_{1}\left[w e^{R_{2}(w)}\right]-h_{2}(w)+R_{2}(w)\right) \triangleq h_{1}\left[w e^{h_{2}(w)}\right] \backslash .
$$

Also we have

and

$$
\left|w e^{h_{2}(w)}\right|>r \cdot e^{K r n_{2}} \quad\left(w \in O_{1},|w|=r>R_{0}\right)
$$

$$
\left|w e^{h_{2}(w)}\right|<r \cdot e^{-K r n_{2}} \quad\left(w \in O_{2},|w|=r>R_{0}\right)
$$

for some positive constant $K$.

Now assuming that $h_{1}{ }^{+}$is transcendental, we use (4) with $\varepsilon=1 / 2$. Then there exists $\left\{r_{n}\right\}$ such that

$$
m\left(r_{n}, h_{1}^{+}\right) \geqq M\left(r_{n}, h_{1}^{+}\right)^{1 / 2} .
$$

Then we can find an unbounded sequence $\left\{t_{n}\right\}$ of real numbers such that $\left|w \cdot e^{h_{2}(w)}\right|=r_{n}$ for some $w\left(w \in O_{1}\right.$ and $\left.\mid w=t_{n}\right)$. In this case,

$$
\begin{aligned}
\left|h_{1}\left(w \cdot e^{h_{2}(w)}\right)\right| & \geqq m\left(r_{n}, h_{1}^{+}\right)+O(1) \\
& \geqq M\left(r_{n}, h_{1}^{+}\right)^{1 / 2}
\end{aligned}
$$
that

On the other hand, for any natural number $N$, there exists $R_{0}=R_{0}(N)$ such

$$
M\left(R, h_{1}{ }^{+}\right)>R^{N} \quad\left(\text { for } R \geqq R_{0}\right)
$$

because of transcendency of $h_{1}{ }^{+}$. Therefore (11) becomes

$$
\left|h_{1}\left(w \cdot e^{h_{2}(w)}\right)\right| \geqq\left(r_{n}\right)^{N / 2} .
$$

Now by (10), $r_{n}>t_{n} \cdot e^{K \cdot t_{n} n_{2}}$. Hence (noting (9)), we have the inequality

$$
c \cdot t_{n} N_{1} \cdot e^{c^{\prime} N_{1} t_{n} N_{2}}>t_{n}{ }^{N / 2} \cdot e^{(1 / 2) N K t_{n} n_{2}} \quad\left(n \geqq n_{0}\right)
$$

for some constants $c$ and $c^{\prime}(>0)$. This contradicts $n_{2} \geqq N_{2}$ and the arbitrariness of $N$. And hence $h_{1}{ }^{+}$must be a polynomial.

We can prove that $h_{1}^{-}(1 / w)$ must be a polynomial in the similar way. 
Hence we deduce that $h_{1}$ is a rational function, as is to be proved.

Finally we prove that both sides of (3) are constants. Putting $h_{2}(w)-R_{2}(w)$ $-G(w)$ and assuming that $G(w)$ is a non-constant rational function, then we have

$$
G(w)=-h_{1}\left[w e^{R_{2}(w)+G(w)}\right]+R_{1}\left[w e^{R_{2}(w)}\right] .
$$

Furthermore let us substitute $w$ by $e^{z}$, then

$$
G\left(e^{z}\right)=-h_{1}\left[e^{z+R_{2}\left(e^{z}\right)+G\left(e^{z}\right)}\right]+R_{1}\left[e^{z+R_{2}\left(e^{z}\right)}\right]
$$

Assuming that $R_{2}(w)+G(w) \not \equiv$ constant, then we can easily show that

$$
\begin{aligned}
& T\left(r, G\left(e^{z}\right)\right)=o\left\{T\left(r, e^{z+R_{2}\left(e^{z}\right)}\right)\right\}, \\
& T\left(r, G\left(e^{z}\right)\right)=o\left\{T\left(r, e^{z+R_{2}\left(e^{z}\right)+G\left(e^{z}\right)}\right)\right\}
\end{aligned}
$$

as $r \rightarrow+\infty$. By Borel's unicity theorem [3], (12) is immpossible, because that $h_{1}(u)$ and $R_{1}(w)$ are rational functions in $w$ whose coefficients are constants.

Next if $R_{2}(w)+G(w) \equiv$ constant, say $c$, then

$$
h_{1}\left[e^{z+R_{2}\left(e^{z}\right)+G\left(e^{z}\right)}\right]=h_{1}\left[e^{z+c}\right] .
$$

Hence (12) is immpossible in the similar way.

Therefore $G(w)$ is a constant, say $K$.

Then by (3),

Hence

$$
\left\{\begin{array}{l}
h_{2}(w)=R_{2}(w)+K \\
h_{1}\left[w \cdot e^{h_{2}(w)}\right]=R_{1}\left[w \cdot e^{R_{2}(w)}\right]-K
\end{array}\right.
$$

$$
h_{1}\left[w \cdot e^{K} \cdot e^{R_{2}(w)}\right]=R_{1}\left[w \cdot e^{R_{2}(w)}\right]-K .
$$

Let $x$ be $w e^{K} e^{R_{2}(w)}$, then we have

$$
h_{1}(x)=R_{1}\left(e^{-K} \cdot x\right)-K \text {. }
$$

By (2), (13) and (14),

Then

$$
\left\{\begin{array}{l}
f(z)=z-K+R_{1}\left(e^{z-K}\right) \\
g(z)=K+z+R_{2}\left(e^{z}\right)
\end{array}\right.
$$

$$
\left\{\begin{array}{l}
f \circ T(w)=w+R_{1}\left(e^{w}\right) \\
T^{-1} \circ g(z)=z+R_{2}\left(e^{z}\right)
\end{array}\right.
$$

with $z=T(w)=w+K$. This completes the proof of our theorem. q. e. d.

Acknowledgement. The auther wishes to thank the referee for many valuable comments and suggestions. 


\section{REFERENCES}

[1 J BAKER, I. N. AND GROSS, F., Further results on factorization of entire functions, Proc. Symp. Pure Math. 11 (1968) 30-35.

[2] GROSS, F., Factorization of meromorphic functions, U.S. Geovernment printing office, Washington D.C. (1972).

[3] NiIno, K. AND OZAWA, M., Deficiencies of an entire algebroid function, Kסdai Math. Sem. Rep. 22 (1970) 98-113.

[4 ] OZAWA, M., Factorization of entire functions, Tóhoku Math. J. 27 (1975) 321-336.

[5] OZAWA, M., On certain criteria for the left-primeness of entire functiins, Kōda1 Math. Sem. Rep. 26 (1975) 304-317.

[6] Pólya, G., On an integral function of an integral function, J. London Math. Soc. 1 (1926) 12-15.

[7] Ritt, J.F., Prime and composite polynomials, Trans. Amer. Math. Soc. 23 (1922) 51-66.

[8] URABE, H., Uniqueniss of factorization under compostion of certain entire functions, J. Math. Kyoto Univ. 18 (No. 1) (1978) 95-120.

DEPARTMENT OF MATHEMATICS, SCIENCE UNIVERSITY OF TOKYO,

NODA, CHIBA, JAPAN. 